Pensamiento Crítico No. 7, pág. 35 - 47

\title{
La Nueva Macroeconomía Clásica Una Versión Algebraica Sencilla \\ Richard Roca
}

Resumen: El presente trabajo expone un modelo sencillo que recoge los fundamentos básicos del enfoque de la nueva macroeconomía clásica con expectativas racionales.

Palabras claves: Macroeconomía, Nuevos Clásicos, expectativas racionales, modelos económicos.

\section{INTRODUCCIÓN}

Esta teoría fue desarrollada por Tomas Sargent, Niel Wallace, Robert Barro liderados por Robert Lucas quien introdujo la hipótesis de expectativas racionales a la macroeconomía. Sostiene que los ciclos económicos se deben básicamente a los cambios sorpresivos de la política económica, especialmente de la política monetaria. 


\section{Richard Roca}

Este enfoque trata de explicar las fluctuaciones económicas partiendo de sólidos fundamentos microeconómicos suponiendo que los mercados son competitivos, los precios son completamente flexibles, los agentes son optimizadores: los consumidores maximizan utilidad y los empresarios maximizan beneficios. Además esta teoría usa la hipótesis de expectativas racionales desarrollada por John Muth (1961) la cual se caracteriza por el uso eficiente de la información, lo que implica que los errores de predicción no sean sistemáticos ni predecibles, y que además, sean los menores posibles. De acuerdo con la hipótesis de expectativas racionales sería tonto que los agentes económicos desperdicien la información disponible por lo que sus pronósticos se hacen usando toda la información relevante disponible en el momento que se hace el pronóstico:

$$
{ }_{t} P_{t+1}^{e}=E\left[P_{t+1} \mid \text { Informacion }_{t}\right]
$$

Lo cual se suele expresar también como: $\quad{ }_{t} P_{t+1}^{e}=\underset{t}{E} P_{t+1}$

La información disponible incluye los valores pasados de las variables endógenas y exógenas además de la teoría económica relevante. Lo que implica que los agentes formen sus expectativas de la misma manera que lo haría la teoría económica.

\section{VERSIÓN ALGEBRAICA DE LOS NUEVOS CLÁSICOS}

Seguidamente veamos una versión algebraica simple de esta teoría.

\subsection{La Curva de Oferta Agregada de Lucas}

Supongamos una función de Producción neoclásica Cobb-Douglas con rendimientos a escala constante y rendimientos marginales decrecientes:

$$
Y_{t}=\Lambda N_{t}^{\alpha} K_{t}^{1-\alpha}
$$

Donde $Y$ es el nivel de producción, $\Lambda$ es un indicador del nivel tecnológico, $N$ el nivel de empleo, $K$ el stock de capital de la economía. Para que haya rendimientos a escala constantes y a la vez rendimientos marginales decrecientes de los insumos $\alpha$ debe ser positivo y menor a uno.

La demanda de trabajo sale de maximizar los beneficios para lo cual el producto marginal de trabajo $(P M N)$ se debe igualar al salario real efectivo $(W / P)$ ya que las empresas conocen tanto el salario nominal que pagan a los trabajadores y el nivel de precios de los bienes finales pues ellas los venden: 
La nueva macroeconomía clásica - una versión algebraica sencilla

$$
P M N_{t}=\frac{W_{t}}{P_{t}}
$$

Derivando la función de producción respecto al trabajo y reemplazándola en la (2):

$$
\alpha \Lambda N_{t}^{\alpha-1} K_{t}^{1-\alpha}=\frac{W_{t}}{P_{t}}
$$

La oferta de trabajo depende directamente del salario real esperado $\left(W_{t} / P_{t}^{e}\right)$. Se supone que los trabajadores conocen el salario nominal del periodo pero no el nivel de precios por lo que no se conoce el poder de compra de sus salarios, pero, dado que lo que interesa es el poder de compra forman una expectativa sobre el nivel de precios actual. Además, la oferta de trabajo depende inversamente de la preferencia por el descanso $(\ell)$ :

$$
N_{t}^{S}=\frac{1}{\ell} \frac{W_{t}}{P_{t}^{e}}
$$

Considerando que los salarios nominales son totalmente flexibles de tal forma que en cada periodo se equilibra el mercado laboral $\left(N_{t}^{S}=N_{t}^{d}=N_{t}\right)$ de (3) y (4) se obtiene la función del empleo de equilibrio de corto plazo:

$$
N_{t}=\left(\frac{\alpha \Lambda K_{t}^{1-\alpha}}{\ell} \frac{P_{t}}{P_{t}^{e}}\right)^{\frac{1}{2-\alpha}}
$$

Reemplazándolo en la función de producción (1) se obtiene la función de oferta agregada:

$$
Y_{t}^{S}=\alpha^{\frac{\alpha}{2-\alpha}} \Lambda^{\frac{2}{2-\alpha}} K_{t}^{\frac{2(1-\alpha)}{2-\alpha}} \ell^{\frac{-\alpha}{2-\alpha}}\left(\frac{P_{t}}{P_{t}^{e}}\right)^{\frac{\alpha}{2-\alpha}}
$$

Teniendo en cuenta que cuando el nivel de precios efectivo es igual al esperado el nivel de producción efectivo y potencial $(\psi)$ son iguales la ecuación anterior se puede escribir como:

$$
Y_{t}^{S}=\psi\left(\frac{P_{t}}{P_{t}^{e}}\right)^{\frac{\alpha}{2-\alpha}}
$$

La ecuación (6) es una versión simple de la famosa función de Oferta Agregada de Lucas de corto plazo, la cual nos muestra que, dado el nivel de producción potencial y las expectativas del nivel de precios, hay una relación directa entre el nivel de precios efectivo y el nivel de producción ofrecido. Al incrementarse el nivel de precios, sin que se modifique las expectativas de precios, a las empresas les conviene contratar más trabajadores y producir más.

A corto plazo, un aumento del nivel de precios de $P_{1}$ a $P_{2}$ conocido por los empresarios, pero no 


\section{Richard Roca}

por los trabajadores, lleva a un aumento del nivel de producción ofrecido. En dicho caso aumenta la demanda de trabajo lo que eleva los salarios nominales pero en menor proporción de lo que aumenta el nivel de precios por lo que el salario real efectivo se reduce. Sin embargo, los trabajadores al observar el aumento del salario nominal creen que sus salarios reales han mejorado, sufren una mala percepción monetaria, y aceptan trabajar más horas, punto 2 en el mercado de trabajo, con lo que se tiene un mayor nivel de empleo y un mayor nivel de producción ofrecido. La curva de oferta agregada a corto plazo tiene pendiente positiva como se muestra en la figura 1.

Cambios del nivel de precios esperado, sin que se modifique el nivel de precios efectivo, desplazan la curva de oferta de trabajo afectando el nivel de empleo por lo que cambia el nivel de producción ofrecido desplazando la curva de oferta agregada de manera similar al caso de la teoría monetarista solo que los cambios de las expectativas de precios no se dan de manera adaptativa sino por un cambio del conjunto de información.

\section{Figura 1.}

\section{La Curva de Oferta Agregada de Lucas de Corto Plazo}

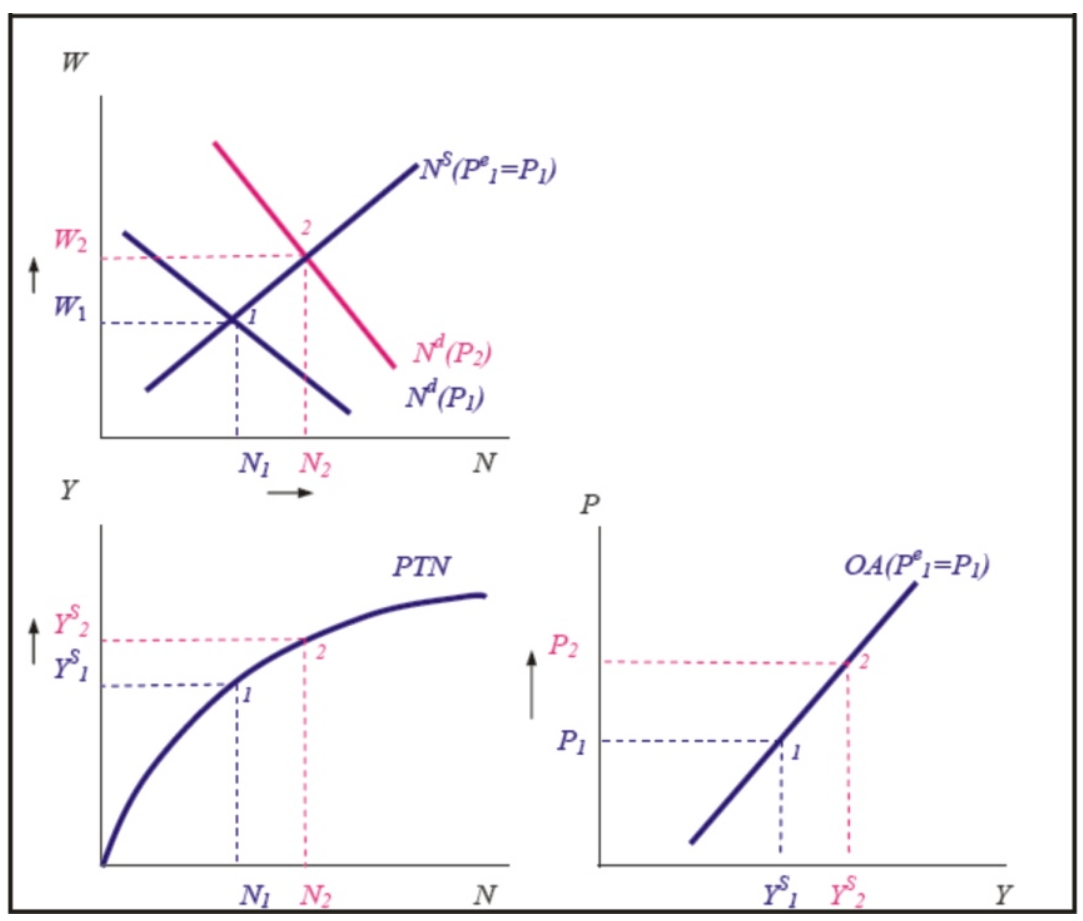




\section{La nueva macroeconomía clásica - una versión algebraica sencilla}

\section{La Demanda Agregada}

La función de la Demanda Agregada de bienes y servicios finales es similar a la considerada en el Monetarismo Moderno:

$$
Y_{t}^{d}=\gamma A_{t}+\Phi \frac{M_{t}}{P_{t}}
$$

Dado que el nivel de precios se considera completamente flexible este se ajusta rápidamente de modo que en cada periodo se igualan el nivel de producción ofrecido y demandado:

$$
\begin{gathered}
Y_{t}^{S}=Y_{t}^{d} \\
Y^{P(}\left(\frac{P_{t}}{P_{t}^{e}}\right)^{\frac{\alpha}{2-\alpha}}=\gamma A+\Phi \frac{M}{P}
\end{gathered}
$$

En el equilibrio de largo plazo las expectativas deberían ser correctas:

$$
P^{e}=P
$$

Por lo que a largo plazo el equilibrio es con pleno empleo:

$$
Y^{S}=\psi=Y^{d}
$$

Combinando (10) con (7) se obtiene el nivel de precios de largo plazo:

$$
\begin{gathered}
\psi=\gamma A+\Phi \frac{M}{P} \\
P=\frac{\Phi M}{\psi-\gamma A}
\end{gathered}
$$

De donde se deduce que, si no se modifica ni el nivel de producción de pleno empleo $(\psi)$ ni la demanda agregada autónoma $(A)$, el nivel de precios de largo plazo es proporcional a la cantidad nominal de dinero lo que coincide con lo planteado por la teoría cuantitativa pero a largo plazo cuando las expectativas son correctas.

A corto plazo, en cualquier periodo las expectativas el nivel de precios esperado para el periodo $t$ por los trabajadores, que no tienen toda la información pero si conocen el modelo económico relevante, esperan el nivel de precios que el mismo modelo pronosticaría por lo que de la solución del nivel de precios tomando expectativas: 


\section{Richard Roca}

$$
P_{t}^{e}=\frac{\Phi M_{t}^{e}}{\psi^{e}-\gamma A_{t}^{e}}
$$

Reemplazándolo en la ecuación (6):

$$
Y_{t}^{S}=\psi\left(\frac{P_{t}\left(\psi^{e}-\gamma A_{t}^{e}\right)}{\Phi M_{t}^{e}}\right)^{\frac{\alpha}{2-\alpha}}
$$

La que es la ecuación de la curva de oferta de Lucas de corto plazo de esta versión algebraica simple. La oferta agregada estará afectada por las expectativas sobre la cantidad de dinero, el nivel de producción potencial, la demanda autónoma todo lo cual a su vez de la información disponible en el momento en que se forma las expectativas.

Si no hay cambios tecnológicos, ni cambia la dotación de recursos, si se conoce con un retraso de un periodo la cantidad de dinero y el gasto autónomo $A$ y se espera que en el periodo actual la cantidad de dinero sea igual al que se dio en el periodo anterior:

$$
P_{t}^{e}=\frac{\Phi M_{t-1}}{\psi-\gamma A_{t-1}}
$$

Por lo que cambios de la cantidad de dinero de un periodo, si se da sin que se enteren los trabajadores en el mismo periodo pero si en el periodo siguiente, afectaría a las expectativas de nivel de precios con un periodo de retraso afectando a la oferta agregada también con retraso:

$$
Y_{t}^{S}=\psi\left(\frac{P_{t}}{\frac{\Phi M_{t-1}}{\psi-\gamma A_{t}}}\right)^{\frac{\alpha}{2-\alpha}}
$$

Por tanto, a corto plazo, si los precios y salarios son totalmente flexibles, aunque no se tenga toda la información, en cada periodo el equilibrio del mercado de bienes:

$$
\begin{gathered}
Y_{t}^{S}=Y_{t}^{D} \\
\psi\left(\frac{P_{t}\left(\psi^{e}-\gamma A_{t-1}^{e}\right)}{\Phi M_{t-1}^{e}}\right)^{\frac{\alpha}{2-\alpha}}=\gamma A_{t}+\Phi \frac{M_{t}}{P_{t}}
\end{gathered}
$$

De donde se obtiene el nivel de precios de cada periodo. 
El nivel de producción de cada periodo se obtendría al reemplazar la solución del nivel de precios de cada periodo en la ecuación de la demanda agregada (7). Si no se vuelve a modificar la cantidad de dinero, en el subsiguiente periodo, no se modificaría las expectativas de precios las que a su vez serian acertadas por lo que el nivel de precios ofrecido seria igual al de pleno empleo.

\section{El Equilibrio General}

Dado que precios y salarios son totalmente flexibles a largo plazo se tiende al pleno empleo. A diferencia del Monetarismo Moderno el ajuste en la teoría de los Nuevos Clásicos es mucho más rápido. A largo plazo, cuando se tenga toda la información, las expectativas no pueden ser equivocadas por lo que el nivel de precios esperado sería igual al nivel de precios efectivo $P^{e}=P$ igualándose el nivel de producción al de pleno empleo y la tasa de desempleo igual a la tasa natural de desempleo.

Figura 2.

Equilibrio General Nuevo Clásico

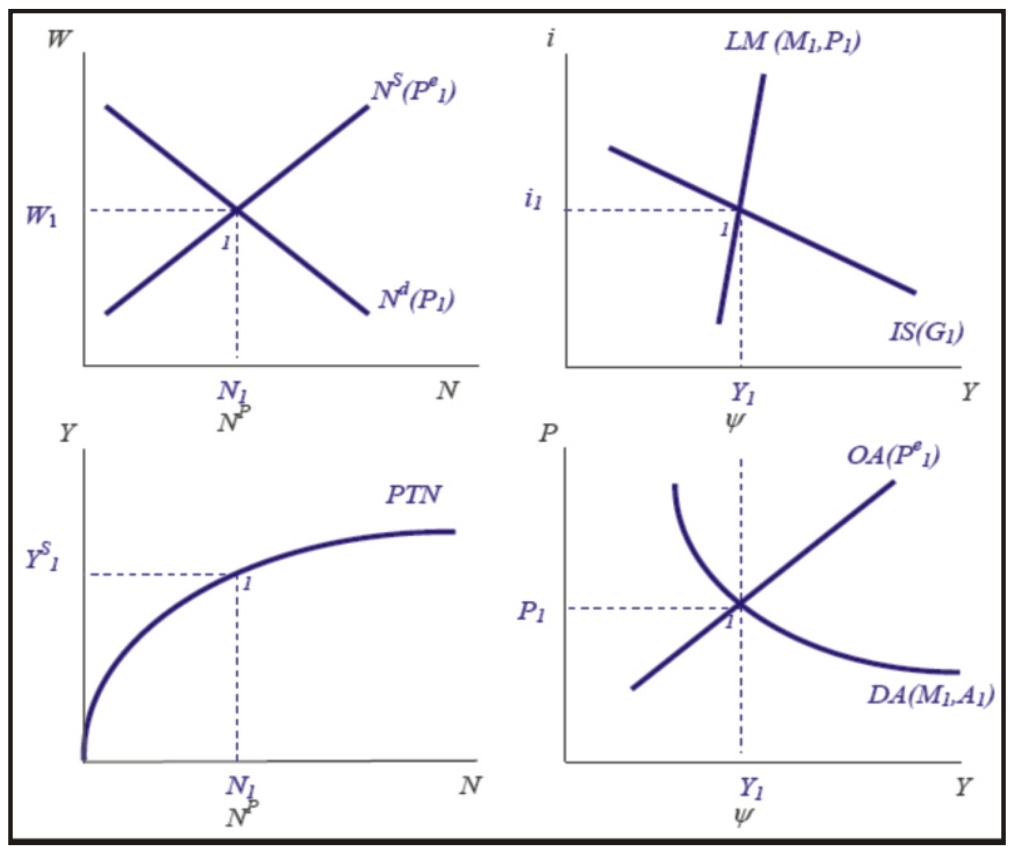




\section{Richard Roca}

\section{Política Monetaria Sorpresiva}

Supongamos que la economía esta en equilibrio en el periodo1, punto 1 de la figura 3 , si en el periodo 2 aumenta de la cantidad de dinero de manera sorpresiva desplazaría la curva $L M$ a la derecha lo que desplazaría a su vez la curva $D A$ a la derecha pasando por el punto . Ello genera un exceso de demanda agregada. El nivel de precios sube a $P_{2}$ lo que incrementa la demanda de trabajo, aumenta los salarios nominales, pero en menor proporción que el nivel de precios por lo que cae el salario real. Los trabajadores creerían que ha subido el salario real por lo que están dispuestos a trabajar más y el nivel del empleo sube a $N_{2}$ y aumenta el nivel de producción a $Y_{2}$. Pero en el periodo 3 los trabajadores se enterarían del aumento de la cantidad de dinero y aumentan sus expectativas de precios por lo que se trasladaría la curva de oferta de trabajo a la izquierda. Ello genera un exceso de demanda de trabajo lo que incrementa los salarios nominales y reduce el nivel del empleo reduciéndose el nivel de producción ofrecido. Esto implica un desplazamiento de la curva OA hacia la izquierda lo que aumenta el nivel de Precios en el periodo 3.

Figura 3.

Aumento Sorpresivo de la Cantidad de Dinero

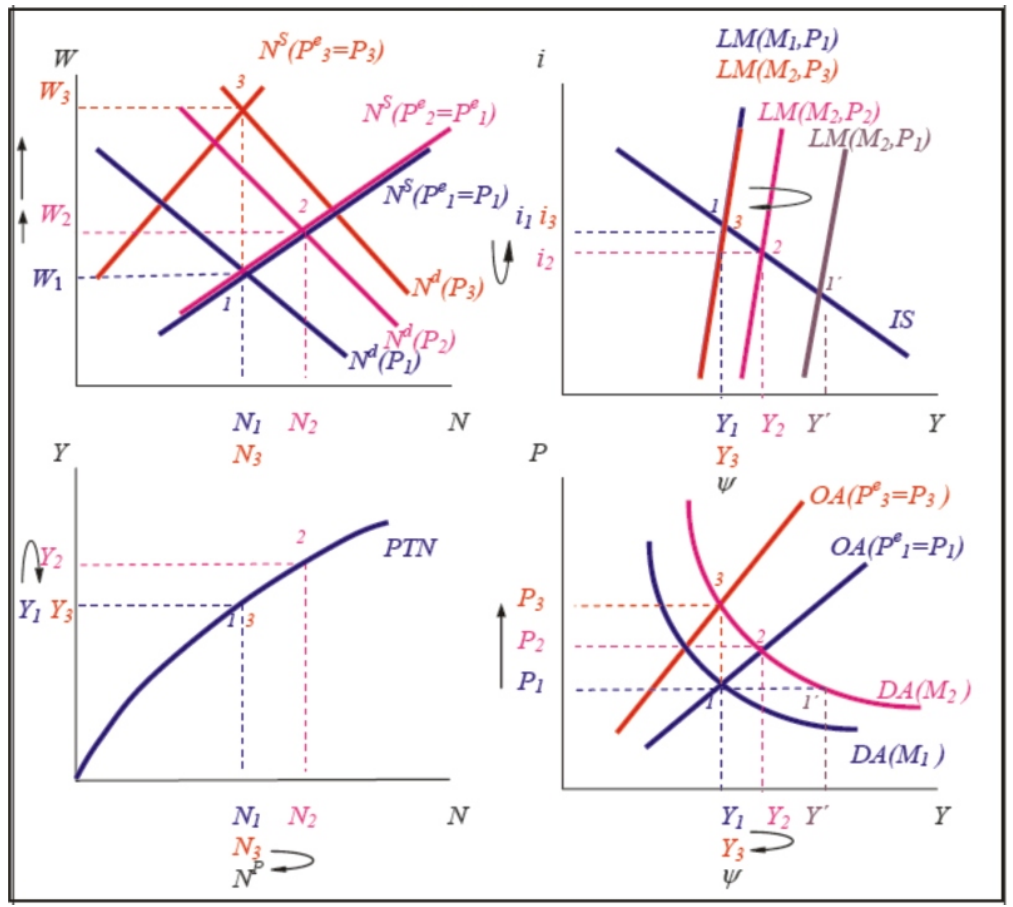


La nueva macroeconomía clásica - una versión algebraica sencilla

\section{Política Monetaria Anunciada}

Supongamos que la economía esta en equilibrio en el periodo1, punto 1 de la figura 4, si en el periodo 2 aumenta de la cantidad de dinero de manera anunciada desplazaría la curva $L M$ a la derecha lo que desplazaría a su vez la curva $D A$ a la derecha pasando por el punto 1'.

Ello genera un exceso de demanda agregada. Simultáneamente los trabajadores enterados del aumento de la cantidad de dinero elevan sus expectativas de precios desplazando a la izquierda tanto la curva de oferta de trabajo como la curva de oferta agregada de Lucas de corto plazo. El exceso de demanda de trabajo eleva los salarios nominales a $W_{2}$ sin que cambie el nivel de empleo $N_{2}=N_{1}$. El exceso de demanda de bienes eleva el nivel de precios a $P_{2}$ sin que cambie el nivel de producción $Y_{2}=Y_{1}=\psi$.

Figura 4.

Aumento Anunciado de la Cantidad de Dinero

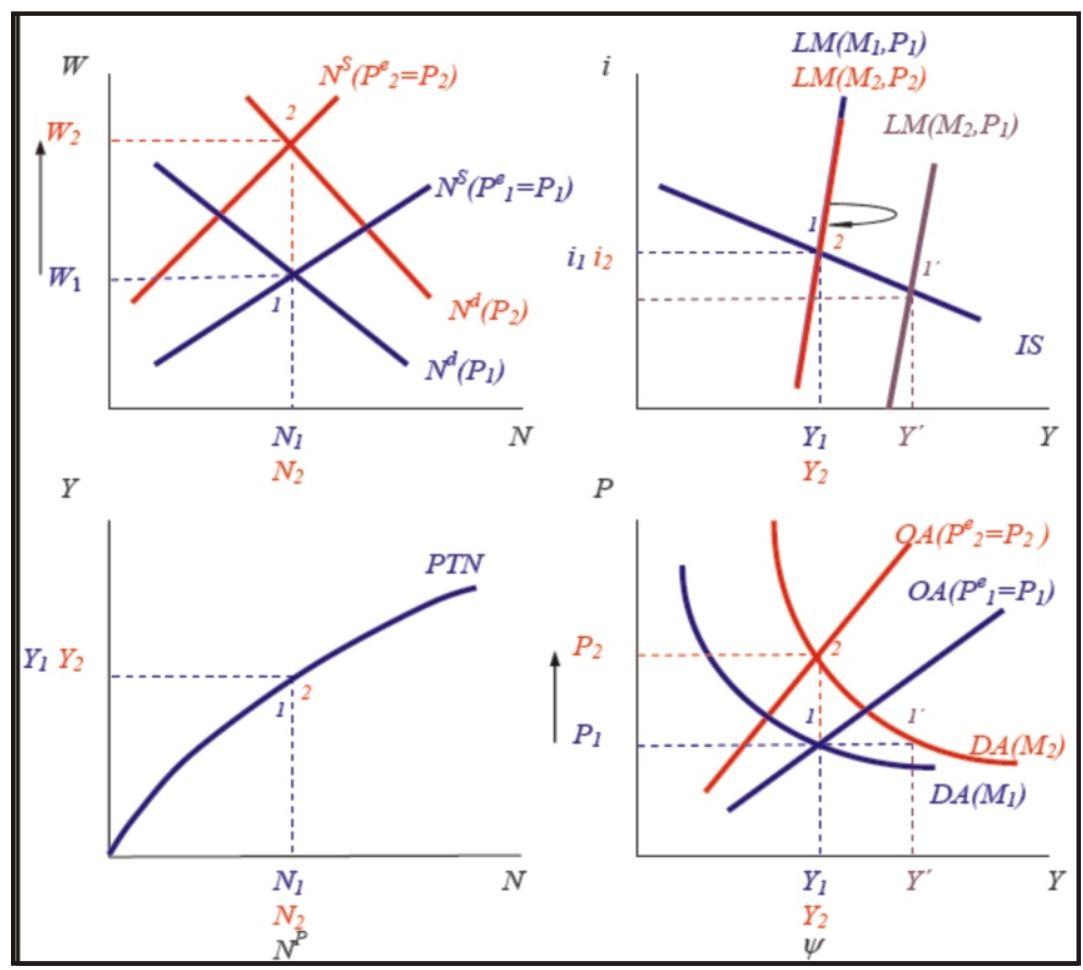

Este caso se conoce como el de la "inefectividad de la política monetaria anunciada" 


\section{Richard Roca}

\section{Caída Inesperada de la Demanda Agregada Autónoma}

Supongamos que la economía esta en pleno empleo en el periodo1, punto 1 de la figura 5, si en el periodo 2 se reduce inesperadamente la inversión autónoma se desplazaría la curva IS a la izquierda lo que desplazaría a su vez la curva $D A$ a la izquierda. Ello genera un exceso de oferta agregada lo que reduce el nivel de precios a $P_{2}$. Ello desplaza la curva de demanda de trabajo en el periodo 2, la curva de oferta de trabajo no se desplaza en el periodo 2 pues los trabajadores no se enteraron la caída de la inversión autónoma. El salario nominal cae a $W_{2}$ y el nivel de empleo a $N_{2}$. En el siguiente periodo los trabajadores se enteran de la caída de la inversión autónoma por lo que esperan un nivel de precios más bajo lo que desplaza a la derecha tanto la curva de oferta de trabajo como la curva de oferta agregada de Lucas de corto plazo. La curva de demanda de trabajo se desplaza algo más a la izquierda por la caída adicional de precios a $P_{3}$. El exceso de oferta de trabajo reduce los salarios nominales a $W_{3}$. El nivel de empleo vuelve al nivel de pleno empleo $N_{3}=N^{P}$. El nivel de producción vuelve al pleno empleo: $Y_{3}=\psi$. Esto implica que no sería necesaria la intervención del gobierno pues el regreso automático al pleno empleo es rápido.

Figura 5.

\section{Caída Inesperada de la Demanda Agregada Autónoma}

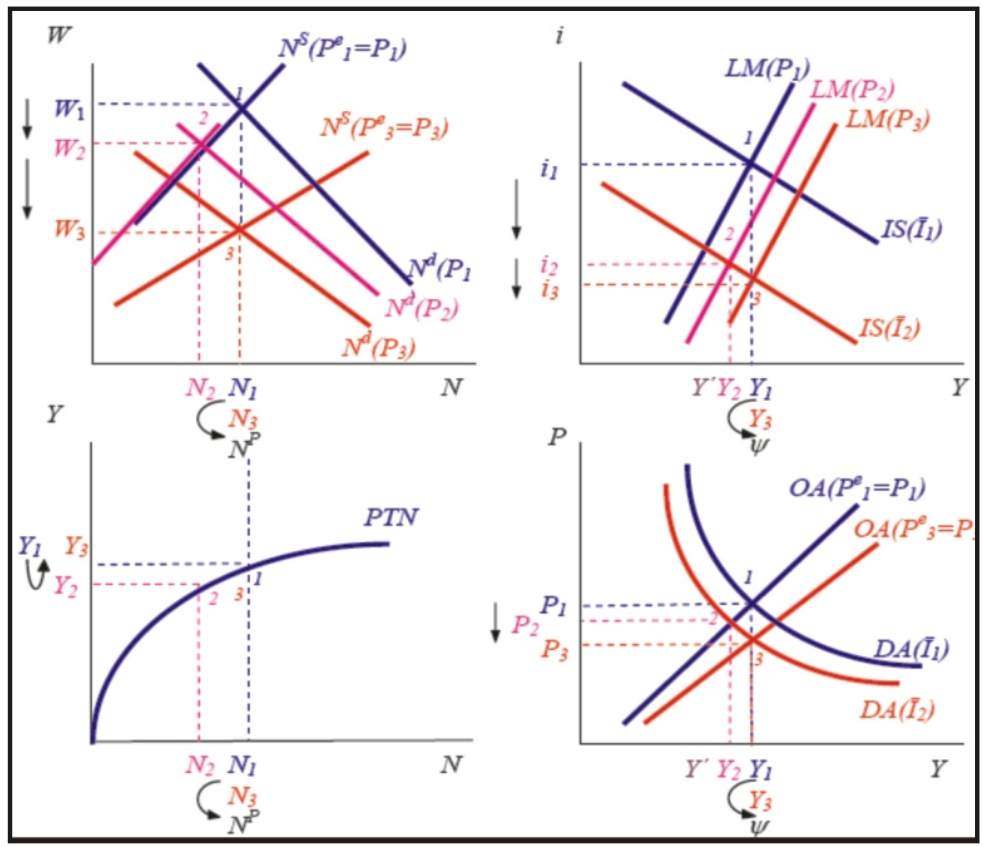


La nueva macroeconomía clásica - una versión algebraica sencilla

\section{Aumento de la Preferencia por el Descanso}

La única forma de que esta teoría muestre una reducción sostenida del nivel del empleo, sin cambios tecnológicos ni reducción de stock de capital, es que se incremente la preferencia por el ocio $(\ell)$. Ello que desplazaría la curva de oferta de trabajo y la curva de oferta agregada a la izquierda. En el mercado de trabajo se eleva el salario real y cae el nivel del empleo. En el mercado de bienes sube el nivel de precios y cae el nivel de producción. En el plano de la IS-LM la tasa de interés se incrementa. Si bien se reduce el nivel del empleo y el nivel de producción de todas maneras se seguiría con pleno empleo. Todo el desempleo es voluntario, como se muestra en la figura 6.

Figura 6.

Aumento de la Preferncia por el Ocio

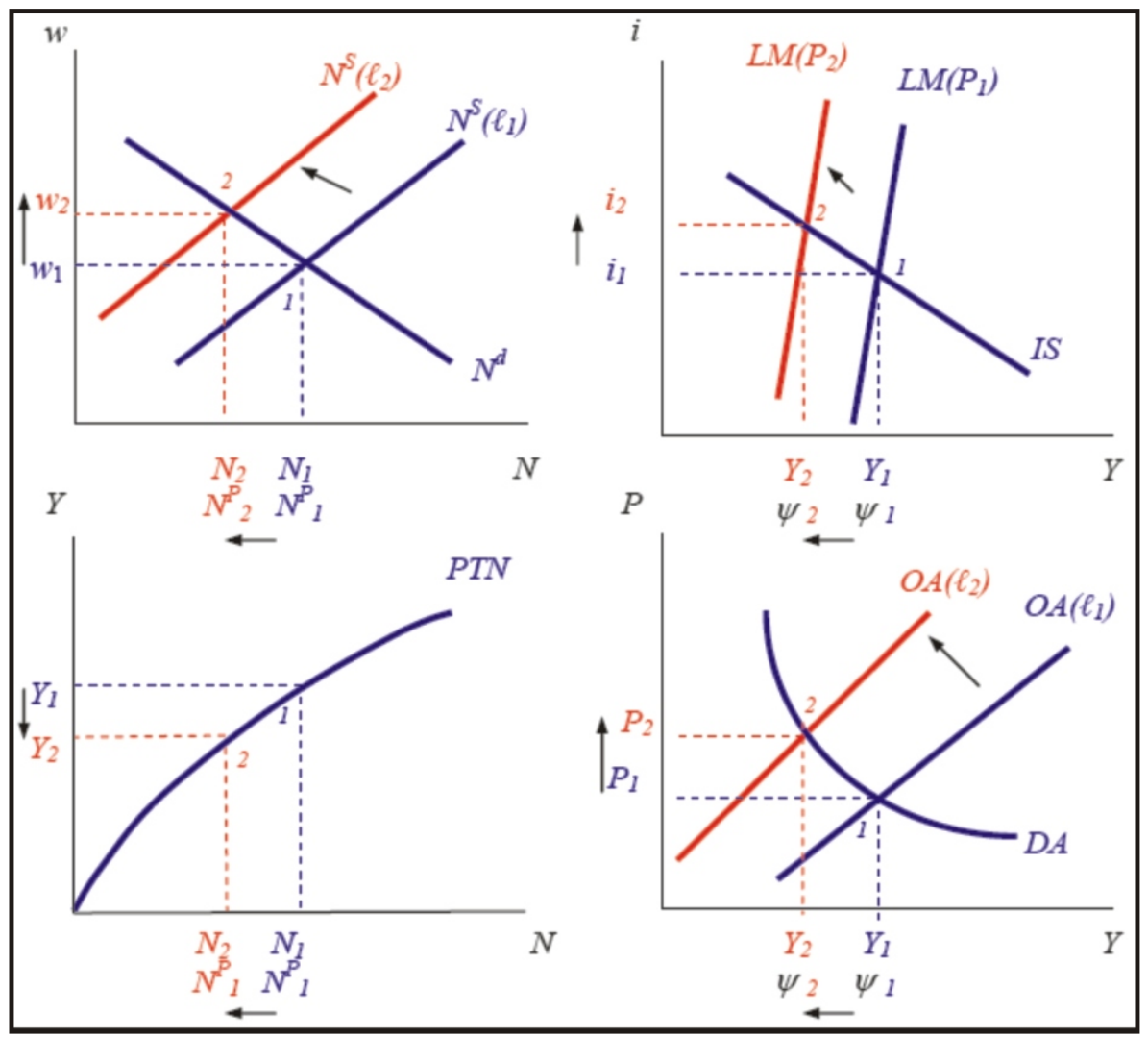




\section{Richard Roca}

\section{CONCLUSIONES}

Estos resultados llevó a los nuevos clásicos a sostener que cambios anunciados de la política monetaria no provocarían cambios del nivel de producción, ni del nivel de empleo, ni la tasa de interés por lo que cambios anunciados del dinero seria neutral inclusive en el corto plazo.

Los aumentos sorpresivos de la cantidad de dinero, enfatizados por Barro (1977) aumentarían el nivel de producción y el nivel del empleo pero solo mientras dure la sorpresa o el engaño. Una vez que los trabajadores se enteren de cual es la verdadera cantidad de dinero en circulación ajustaran sus expectativas de precios volviendo la economía inmediatamente al pleno empleo.

De manera más exacta, las expectativas de precios dependen de la cantidad de dinero que el público cree que va a circular, no necesariamente de la que se observó en el periodo anterior, ni de los anuncios de las autoridades económicas si no tienen credibilidad. La falta de confianza en las autoridades monetarias podrían inducir a pensar que la cantidad de dinero sea mayor llevando a aumentos del nivel de precios aunque no se incremente la oferta monetaria por lo que el tema de la confianza y credibilidad se vuelven importantes.

Adicionalmente dado que en cada periodo los salarios nominales y el nivel de precios son flexibles las cantidades ofrecidas y demandadas de trabajo se igualan por lo que el mercado de trabajo esta con pleno empleo.

Esta teoría afirma que la economía tendría pleno empleo de manera automática por lo que el gobierno no necesita aplicar políticas activas ni discrecionales para llevar a la economía al pleno empleo. Dado que los desvíos del nivel de producción con respecto al potencial se explican por la falta de información bastaría con que el gobierno, en un mensaje a la nación, de información a los agentes económicos para que la economía vuelva al pleno empleo lo que suena muy poco convincente.

Estos resultados de que solo las sorpresas monetarias explicarían los ciclos económicos y que no hay desempleo involuntario recibieron fuertes críticas. Para superar dichas deficiencias desde la misma orilla neoclásica se desarrolló la llamada teoría de los ciclos reales pasando el centro de atención a los shocks tecnológicos para explicar las fluctuaciones económicas. Desde el frente keynesiano se desarrolló una nueva vertiente conocida como los Nuevos Keynesianos quienes aceptaron la hipótesis de expectativas racionales pero 


\section{La nueva macroeconomía clásica - una versión algebraica sencilla}

mantuvieron las rigideces keynesianas dándoles fundamentos microeconómicos en un contexto de competencia imperfecta.

\section{BIBLIOGRAFÍA}

Barro, R. (1976) "Rational Expectations and the Role of Monetary Policy." Journal of Monetary Economics 2. Enero, p.1-32.

Barro, R. (1977) "Unanticipated Money Growth and Unemployment in the United States". Barro, R. (1979) "Second Thoughts on Keynesian Economics". Barro, R. (1984) "Rational Expectations and Macroeconomics in 1984".

Lucas, R. (1972) "Econometric Testing of the Natural Rate Hypothesis", en O. Eckstein, editor, Econometrics of Price Determination. Washington, DC: Board of Governors of the Federal Reserve System. Reimpreso en Lucas, 1981.

Lucas, R. (1972) "Expectations and the Neutrality of Money". Journal of Economic Theory, Vol. 4 (2), p.10324. Reimpreso en Lucas, 1981.

Lucas, R. (1973) "Some International Evidence on Output-Inflation Trade-Offs", American Economic review, Vol.63 (3), p.326-34. Reimpreso en Lucas, 1981.

Lucas, R. (1976) "Econometric Policy Evaluation: A critique", Carnegie-Rochester Conference Series on Public Policy, Vol. 1, p.19-46. Reimpreso en Lucas, 1981.

Lucas, R. (1981) Studies in Business Cycle Theory. 2nd Edition, 1991. Cambridge, Mass: The M.I.T. Press.

Lucas, R. and E.C. Prescott (1974) "Equilibrium Search and Unemployment", Journal of Economic Theory, Vol. 7, p.188-209.

Lucas, R. y T. Sargent (1979) "After Keynesian Macroeconomics", en After the Phillips Curve: Persistence of high inflation and high unemployment. Boston: Federal Reserve.

Lucas, R. y Rapping, L. (1969) "Real wages, employment and inflation": Journal of Political Economy, 77.

Lucas, R. 1980. "Two Illustrations of the Quantity Theory of Money," American Economic Review, vol. 70(5), pages 1005-14.

Muth, J. (1961) "Rational Expectations and the Theory of Price Movements". Econometrica, Vol. 29 (3), p.31525.

Sargent, T. y Wallace, N. (1976) "Rational expectations and the theory of economic policy". Journal of Monetary economics, 2.

Wallace, N. (1996) "Las expectativas racionales y el fin de la macroeconomía". En Apuntes No. 38. Universidad del Pacífico. 